\title{
Surface Segregation of Ge at SiGe(001) by Concerted Exchange Pathways
}

\author{
P. Bogusławski ${ }^{1,2}$ and J. Bernholc ${ }^{2}$ \\ ${ }^{1}$ Instytut Fizyki PAN, 02-668 Warsaw, Poland \\ ${ }^{2}$ Department of Physics, North Carolina State University, Raleigh, North Carolina 27695 \\ (Received 31 October 2001; published 5 April 2002)
}

\begin{abstract}
The segregation of Ge during growth on $\mathrm{SiGe}(001)$ surfaces was investigated by ab initio calculations. Four processes involving adatoms rather than ad-dimers were considered. The two most efficient channels proceed by the concerted exchange mechanism and involve a swap between an incorporated Ge and a $\mathrm{Si}$ adatom, or between $\mathrm{Si}$ and $\mathrm{Ge}$ in the first and the second surface layers, respectively. The calculated activation energies of $\sim 1.5 \mathrm{eV}$ explain well the high-temperature experimental data. Segregation mechanisms involving step edges are much less efficient.
\end{abstract}

DOI: 10.1103/PhysRevLett.88.166101

The atomic-scale morphology of $\mathrm{Si} / \mathrm{SiGe}$ quantum structures and interfaces is largely determined by preferential surface segregation of Ge. For this reason, various experimental manifestations of segregation have been extensively investigated in the last decade [1-10]. The tendency of Ge to segregate is indeed surprisingly strong: after a deposition of about $50 \mathrm{ML}$ of $\mathrm{Si}$ on a layer of $\mathrm{SiGe}$, the surface is still covered by about $25 \%$ of $\mathrm{Ge}$ $[3,4]$. The characteristic fingerprints of the segregation are the profiles of $\mathrm{Ge}$ composition close to the $\mathrm{Si} / \mathrm{SiGe}$ and $\mathrm{SiGe} / \mathrm{Si}$ interfaces, which differ substantially from the chemical composition of the deposited flux. These zones characterized by composition gradients achieve thickness of 20-40 A, i.e., several tens of atomic layers [3-6]. In short-period $\mathrm{Si} / \mathrm{Ge}$ superlattices, segregation leads to highly intermixed and asymmetric $\mathrm{Si} / \mathrm{Ge}$ and $\mathrm{Ge} / \mathrm{Si}$ interfaces [8-10]. Finally, surface segregation of Ge has important consequences for the growth of coherent $\mathrm{SiGe}$ islands on $\mathrm{Si}(001)$. This is because the formation and shape of islands are affected by the interplay between misfit strain and surface energy [11], the latter being determined by the chemical composition of the surface. Under typical conditions (slow growth at $500-700{ }^{\circ} \mathrm{C}$ ), the segregation is efficient and surfaces of islands are expected to be $\mathrm{Ge}$ rich almost independently of the actual alloy composition.

In the above experiments, Ge atoms may be regarded as "markers" that reveal atomic-scale features of epitaxial growth, such as the incorporation of deposited atoms and the exchange of atoms between the first few surface layers. This insight is very valuable since a detailed atomistic picture of growth is still lacking. In particular, the mechanism of segregation is not known. Intermixing between Ge adatoms deposited on $\mathrm{Si}(001)$ and surface atoms is observed already at $20-60{ }^{\circ} \mathrm{C}[12,13]$. The intermixing may occur by the exchange of sites between an atom forming an ad-dimer and an incorporated atom with the energy barrier $\gtrsim 1 \mathrm{eV}$ [13]. Ab initio calculations [14] indicate that intermixing with a very small barrier of $0.1 \mathrm{eV}$ may also take place during the last step of formation of an ad-dimer by two Ge adatoms. Analogous intermixing channels involving ad-dimers and characterized by similar barriers
PACS numbers: 68.35.-p, 71.15.Pd, 81.10.Aj

could also lead to segregation of Ge. On the other hand, segregation of $\mathrm{Ge}$ in $\mathrm{SiGe} / \mathrm{Si}$ structures has been modeled by Monte Carlo technique [3]. For $300-800{ }^{\circ} \mathrm{C}$, a good fit to experimental data is obtained with the barrier of $1.4 \mathrm{eV}$, which is much higher than the $0.1 \mathrm{eV}$ obtained in Ref. [14]. Here, we show that segregation and intermixing mechanisms are different at room and at high temperatures, and that the mechanisms dominant at high temperatures do not involve ad-dimers.

Three segregation mechanisms have been proposed so far. They are schematically shown in Fig. 1 as A, B, and C. According to the first [2], segregation takes place on the flat terraces of the surface via an exchange of atoms between the first two surface layers. This concerted exchange $(\mathrm{CE})$ of sites occurs by a rotation of two atoms around the center of their bond. In the second channel [9], segregation is also due to CE events, but they take place at edges of monoatomic steps. Both channels assume that the

(a)

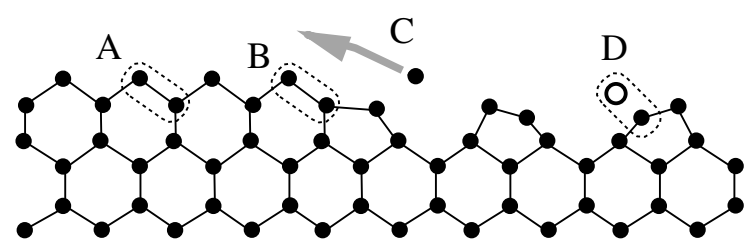

(b)

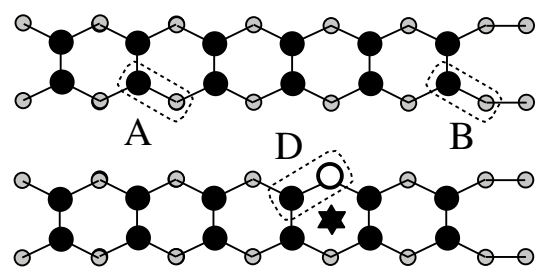

FIG. 1. (a) Side view [projection of atoms on a (110) plane] and (b) top view of the (001) surface with one rebonded monoatomic step $S_{B}$, and four segregation channels: (A) Concerted exchange (CE) at a flat terrace, (B) CE at a step edge, (C) jump over a step edge, and (D) displacive incorporation of a Si adatom. Pairs of atoms exchanging sites are marked with dashes. The white circles for channel D show the equilibrium position of a $\mathrm{Si}$ adatom. See text. 
Ge atoms are already incorporated, i.e., located at substitutional sites of the subsurface layer. The third mechanism [15] assumes that the segregation is due to jumps of $\mathrm{Ge}$ adatoms over step edges from lower to higher terraces. We have also considered a fourth segregation mechanism (D in Fig. 1), in which the incorporation of deposited Si adatoms takes place by $\mathrm{CE}$ with Ge in the surface layer by a "displacive adsorption" [16] (see below for a detailed description of all atomic transformations). Thus far, the efficiency of any of the channels has not been assessed. Furthermore, both misfit strain and differences in bond strengths were considered as the driving forces for segregation [16]. In this Letter, we determine the precise atomistic transformations and activation barriers for each of the above channels by $a b$ initio molecular dynamics and discuss their effect on growth and intermixing at $\mathrm{Si} / \mathrm{Ge}$ interfaces.

In our real-space quantum dynamics simulations $[17,18]$ of CE events, the exchange of sites between the two atoms was performed by using the "adiabatic trajectory" method [20]. In this method, both atoms are rotated with a small, constant speed in a given direction while monitoring the total energy. The atoms are constrained in one direction only: they are free to move in the directions perpendicular to the applied force, thereby enabling the system to find the optimum path. All other atoms are relaxed continuously in response to the forced motion. Importantly, we have also investigated a forced motion of one atom only, obtaining very similar atomic trajectories and energy barriers.

To ascertain the driving force for the segregation, we briefly analyze the surface energetics of SiGe(001). The ground state reconstruction of the (001) surface is $c(2 \times 4)$, in which the surface dimers are tilted and the sign of the tilt alternates along the dimer rows [21]. Because of the structure of the bulk Si lattice, the orientation of the reconstruction is forced to alternate on terraces separated by a single-height step. The side view of this surface, with a single-height step and two tilted dimers on the lower terrace, is shown in Fig. 1. We have considered three locations of a single $\mathrm{Ge}$ atom on $\mathrm{Si}(001)$, namely the "up" and the "down" site of a dimer, and the buried configuration with $\mathrm{Ge}$ in the first subsurface layer. The buried Ge has the highest energy. Moving this atom to the surface provides an energy gain, called the segregation energy $E_{\text {seg }}$, of 0.22 and $0.35 \mathrm{eV}$ for the down and up sites, respectively [22]. Similar values were obtained for the coverage of $0.5 \mathrm{ML}$ of $\mathrm{Ge}$ in the $c(2 \times 4)$ reconstruction. The preferential occupation by $\mathrm{Ge}$ of the up dimer sites has been recently observed [23]. Comparing to misfit energetics, since the strain energy in a SiGe alloy is about $10 \mathrm{meV}$ per atom, an individual segregation event may decrease the strain by this amount at most. This value is much lower than the segregation energy, which demonstrates that segregation is not driven by the release of misfit strain.

We now turn to energy barriers for the different exchanges. In the case of the first segregation channel, one Ge atom initially occupies a buried site in the first subsurface layer at the otherwise pure $\operatorname{Si}(001)$. For the $c(2 \times 4)$ reconstruction, Ge has two nonequivalent nearest neighbors at the surface, which are the up and down atoms from the two adjacent surface dimers. We have considered a few possible paths of $\mathrm{CE}$ for both atoms. For the $\mathrm{CE}$ with the down atom, the trajectory of the $\mathrm{Si}^{*}$-Ge pair (hereafter we use asterisks to denote the host atom involved in CE) and the corresponding total energy changes are shown in Figs. $2 a$ and $2 b$, respectively. In this case, CE was accomplished by forcing the atoms' motion in the [001] direction. We see that the path of CE is more complex than it is assumed in simple models [2]: CE does not involve one but three energy barriers that separate two metastable configurations ( $M_{1}$ and $M_{2}$ in Fig. $2 b$ ) between the initial and the final states. The minima $\mathrm{M}_{1}$ and $\mathrm{M}_{2}$ are higher in energy than the initial configuration by 0.7 and $0.8 \mathrm{eV}$, respectively. As follows from Fig. 2b, the largest barrier involved in $\mathrm{CE}$ is $\mathrm{B}_{1}$, equal to $1.6 \mathrm{eV}$. The final configuration is lower by $0.35 \mathrm{eV}$ than the initial one. In addition, we have considered two other segregation pathways of a buried Ge. The first one is CE with the up Si atom (the left neighbor of Ge in Fig. 2a). This case is similar to the CE with the down atom considered above, since the barrier $\mathrm{B}_{1}$ is $1.75 \mathrm{eV}$ and the final configuration is the same. In the second path, the forced motion was initially along the [110] direction. This pathway is not effective, however, since this time three atoms participate in the event and the energy barrier is about two times higher than in the cases above.

The second segregation channel has been proposed in order to explain both the segregation of Ge and the spontaneous growth of ordered $\mathrm{SiGe}$ alloys [9]. It proceeds via $\mathrm{CE}$ at step edges. At the $\mathrm{Si}(001)$ surface, two types of single-height steps exist [24], $S_{A}$ and $S_{B}$, in which the
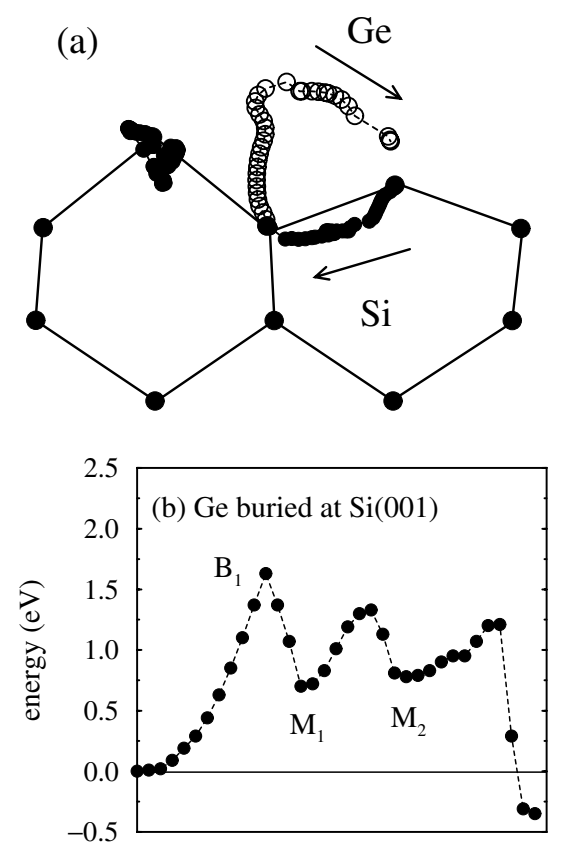

FIG. 2. Concerted exchange of buried Ge at $\mathrm{Si}(001)$ : (a) Side view of the trajectory; (b) the corresponding total energy changes. 
dimer rows on the upper terrace are oriented parallel and perpendicular to the step edge, respectively. There are two types of $S_{B}$ steps: with and without rebonded atoms on the lower edge. In principle, segregation may occur at all three types of edges. However, since the local configuration of atoms at $S_{A}$ is very similar to that at the flat terrace, one does not expect major differences between these two cases. Furthermore, segregation at nonrebonded $S_{B}$ does not lead to energy gain, since the numbers of broken and saturated $\mathrm{Si}$ and Ge bonds remain the same after CE. Segregation is thus expected to occur only for the rebonded $S_{B}$ step, since in this case the segregation energy is nonvanishing [25]. We have tried a number of possible pathways, with the applied forces oriented parallel to either [001] or [110] directions. In all cases, the energy barriers were at least $3 \mathrm{eV}$, i.e., twice as large as in the case of the first channel. The high stiffness of the rebonded step edge is due to it being highly strained, since a segregation event requires the breakage of a bond between Ge and its Si neighbor from the lower terrace. Moreover, the efficiency of this channel is much lower than the previous one, because the number of sites at step edges is much lower than the number of sites at flat terraces.

In the third channel, proposed in Ref. [15] and schematically shown as $C$ in Fig. 1, segregation results from jumps of Ge adatoms over step edges from the lower to the upper terraces (hereafter called up jumps, in contrast to the reverse down jumps). Based on the experimental data and our results we argue that the efficiency of this mechanism is low. We first observe that the actual processes of growth are more complex than it is suggested in Ref. [15]. In particular, during the epitaxy the up and the down jumps of adatoms coexist, and the two fluxes are in equilibrium under steady state conditions. The dominance of the up flux would lead to surface roughening during growth, but this was not reported [1-10]. Next, as was mentioned before, segregation takes place during growth of a mixed SiGe layer. In this case, both $\mathrm{Si}$ and $\mathrm{Ge}$ adatoms jump to the upper terrace, but only the latter process can lead to Ge segregation. Therefore, this mechanism is operative only if the upward flux of Ge is larger than that of $\mathrm{Si}$. This could be due to a lower barrier for the up jumps of Ge relative to that of Si adatoms. However, our calculations do not support this possibility: the calculated barriers for $\mathrm{Si}$ and Ge are the same within $0.1 \mathrm{eV}$. Based on this result we cannot rule out this channel completely, since a difference of $0.1 \mathrm{eV}$ in favor of $\mathrm{Ge}$ can still make the segregation possible, albeit weak. However, this should lead to preferential accumulation of $\mathrm{Ge}$ at the higher terraces of islands, while experiments $[16,23]$ indicate a uniform distribution of $\mathrm{Ge}$ at the surface.

In the last channel (D in Fig. 1) the segregation of Ge occurs by a replacement of a $\mathrm{Si}$ adatom by a Ge adatom. We have examined this displacive incorporation for a $\mathrm{Si}$ adatom on $\mathrm{Ge}(001)$. The equilibrium position of the $\mathrm{Si}$ adatom is situated in between two dimer rows (shown by a white circle in Fig. 1); this location is close to that of a $\mathrm{Si}$ adatom on $\mathrm{Si}(001)$ [26]. The trajectories of the exchanging atoms and the changes in the total energy are shown in Fig. 3. The CE proceeds through two metastable states: A barrier of $1.5 \mathrm{eV}$ separates the initial configuration and the first metastable state $\mathrm{M}_{1}$, and a barrier of $0.3 \mathrm{eV}$ separates $\mathrm{M}_{1}$ and $\mathrm{M}_{2}$. In the latter configuration the segregation is already achieved, because $\mathrm{Si}$ occupies the substitutional site initially occupied by $\mathrm{Ge}^{*}$ and $\mathrm{Ge}^{*}$ becomes an adatom located between two dimers; its position is shown by a star in Fig. 1b, and by a dashed arrow in Fig. 3a. Accordingly, the configuration $\mathrm{M}_{2}$ is lower by $0.15 \mathrm{eV}$ than the initial one. However, an additional $0.1 \mathrm{eV}$ can be gained by forcing the $\mathrm{Ge}^{*}$ adatom to occupy a site between two dimer rows, close to the position initially occupied by the $\mathrm{Si}$ adatom. The last step involves a barrier of $0.6 \mathrm{eV}$. [Very similar results are obtained for the $\mathrm{Si}$ adatom on $\mathrm{Si}(001)$, where the position between the dimer rows is lower by $0.1 \mathrm{eV}$ than the one between two dimers, and the corresponding barrier is also $0.6 \mathrm{eV}$ [26].] We note that both the segregation energies and the barriers for this and the first channel are very close.

How do these results compare with published experimental data? Clearly, a direct comparison with experiment is not possible, since the measured quantity is the distribution of $\mathrm{Si}$ and $\mathrm{Ge}$ atoms close to the surface or the interfaces, and not the segregation energy or the barriers. Previous workers fitted the measured profiles in Monte Carlo simulations of growth, using an atomic-scale model of segregation with $E_{\text {seg }}$ and $E_{b}$ as two free parameters [2-4] and including only the first of the channels considered here. This approach leads to $E_{\text {seg }}=0.28 \pm 0.12$ [2,3(a)], 0.24 [4], or $0.33 \mathrm{eV} \mathrm{[7],} \mathrm{and} E_{b}=1.63 \pm 0.1 \mathrm{eV}$
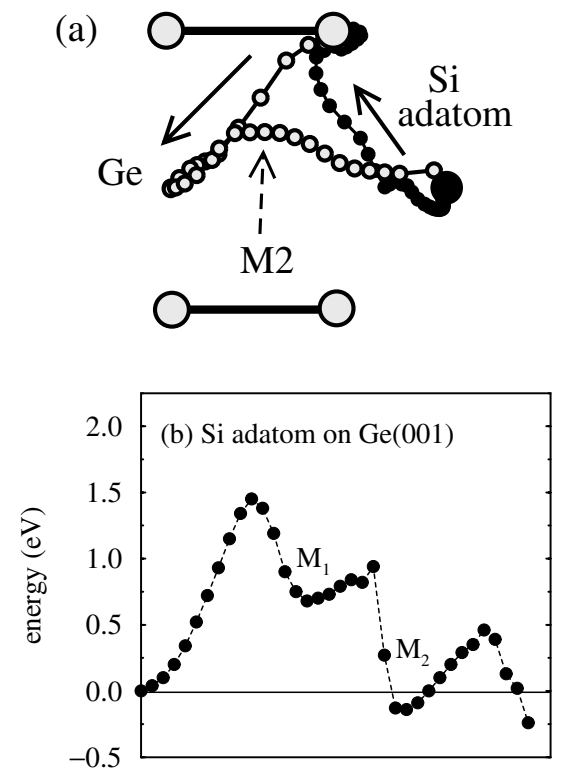

FIG. 3. Concerted exchange of a $\mathrm{Si}$ adatom at $\mathrm{Ge}(001)$ : (a) Top view of the trajectory; the initial positions of $\mathrm{Si}(\mathrm{Ge})$ and their trajectories are shown by large and small black (gray) circles; (b) the corresponding total energy changes. 
[2,3(a)]. In Ref. [3(b)], the Monte Carlo model was modified to include surface diffusion, giving $E_{\mathrm{seg}}=0.13 \mathrm{eV}$ and $E_{b}=1.4 \mathrm{eV}$. Given all implicit and explicit simplifications of the Monte Carlo approach, our values of $E_{\mathrm{seg}} \simeq 0.3 \mathrm{eV}$ and $E_{b} \simeq 1.5 \mathrm{eV}$ are in satisfactory agreement with the experimental data.

Finally, we compare efficiency of the channel D with that of the mechanism involving formation of ad-dimers proposed by Ko et al. [14]. A Si adatom deposited on $\mathrm{Ge}(001)$ at the temperature $550{ }^{\circ} \mathrm{C}$ will exchange sites with an incorporated Ge via the channel D within $\tau_{\mathrm{ex}}=$ $\nu^{-1} \exp \left(E_{b} / k_{B} T\right) \approx 10^{-4} \mathrm{sec}$, where $\nu=10^{13} \mathrm{sec}^{-1}$ is the attempt frequency. The mean distance covered by the adatom within $\tau_{\mathrm{ex}}$ is of the order of $l_{\mathrm{ex}} \approx a\left[\nu \tau_{\mathrm{ex}} \times\right.$ $\left.\exp \left(-E_{\text {diff }} / k_{B} T\right)\right]^{1 / 2}$, where $a$ is the surface lattice constant. Surface diffusion of adatoms is highly anisotropic [26], and the energy barrier for the fast diffusion along the dimer rows is $E_{\mathrm{diff}}=0.6 \mathrm{eV}$. This gives $l_{\mathrm{ex}} \simeq 500 a$. (Diffusion perpendicular to dimer rows may be neglected, and thus the process is one dimensional.) For the typical growth rate of $1 \AA / \mathrm{sec}$, the average number of atoms deposited during $\tau_{\mathrm{ex}}$ within the distance $l_{\mathrm{ex}}$ is 0.04 , which is very small. Consequently, the $\mathrm{Si}$ adatom may exchange sites with an incorporated Ge many times before it meets another adatom and form an ad-dimer. In other words, channel D is more efficient than the mechanism of Ko et al. Similarly, at these growth conditions an incorporated Ge may perform $10^{4} \mathrm{CE}$ events and segregate by channel A before it gets covered by the incoming flux. On the other hand, at temperatures lower than about $350{ }^{\circ} \mathrm{C}$, the mechanism proposed by Ko et al. becomes dominant. (Because of the exponential dependence of the results on energy barriers and growth temperature, more accurate estimates are not possible).

In summary, surface segregation of Ge during growth at $\mathrm{SiGe}(001)$ has been investigated theoretically using quantum molecular dynamics. Four segregation processes were examined: (i) at flat terraces, (ii) at step edges, (iii) via jumps of adatoms over step edges, and (iv) via displacive incorporation of Si adatoms. The lowest energy barrier for segregation, $1.5 \mathrm{eV}$, is associated with the last channel. In competition with it is the first channel, with a barrier of $1.6 \mathrm{eV}$. In both cases, the segregation is found to proceed via a concerted exchange of atoms between the free surface and the first subsurface layer, and both channels are active at typical growth temperatures. These ab initio results explain and reconcile a multitude of experimental data and are in very good agreement with barriers for $\mathrm{Si} / \mathrm{Ge}$ exchange extracted from these experiments.

This work was supported in part by Grant No. KBN 2-P03B-047-19, U.S. DOE and ONR, and a Grant of computer time from the DOD High Performance Computing Modernization Program at NAVO HPC and NCSC.

[1] P. C. Zalm et al., Appl. Phys. Lett. 55, 2520 (1989).
[2] S. Fukatsu et al., Appl. Phys. Lett. 59, 2103 (1991).

[3] (a) D. J. Godbey and M. G. Ancona, Appl. Phys. Lett. 61, 2217 (1992); (b) Surf. Sci. 395, 60 (1998).

[4] Y. Li, G. G. Hembree, and J. A. Venables, Appl. Phys. Lett. 67, 276 (1995).

[5] J. A. Floro and E. Chason, Appl. Phys. Lett. 69, 3830 (1996).

[6] G. G. Jernigan, P. E. Thomson, and C. L. Silverstre, Appl. Phys. Lett. 69, 1894 (1996).

[7] A. M. Lam, Y. J. Zheng, and J. R. Engstrom, Appl. Phys. Lett. 73, 2027 (1998).

[8] E. Muller et al., Phys. Rev. Lett. 63, 1819 (1989).

[9] D. E. Jesson, S. J. Pennycook, and J. M. Baribeau, Phys. Rev. Lett. 66, 750 (1991).

[10] N. Ikarashi, K. Akimoto, T. Tasumi, and K. Ishida, Phys. Rev. Lett. 72, 3198 (1994); 75, 185 (1995).

[11] J. Tersoff and F. K. LeGoues, Phys. Rev. Lett. 72, 3570 (1994).

[12] X. R. Qin, B. S. Swartzentruber, and M. G. Lagally, Phys. Rev. Lett. 84, 4645 (2000).

[13] X. R. Qin, B. S. Swartzentruber, and M. G. Lagally, Phys. Rev. Lett. 85, 3660 (2000).

[14] Y.-J. Ko, K.-H. Park, J.S. Ha, and W.S. Yun, Phys. Rev. B 60, 8158 (1999).

[15] J.F. Nutzel and G. Abstreiter, Phys. Rev. B 53, 13551 (1996).

[16] R. M. Tromp, Phys. Rev. B 47, 7125 (1993).

[17] E. L. Briggs, D. J. Sullivan, and J. Bernholc, Phys. Rev. B 52, R5471 (1995); 54, 14362 (1996).

[18] Surface calculations based on the local density approximation were carried out in a slab geometry with 6 atomic layers. The real-space grid density corresponded to an equivalent energy cutoff of $21 \mathrm{Ry}$ for the plane wave basis. The two bottom layers were held fixed at the ideal lattice positions and the bottom layer was saturated with $\mathrm{H}$ atoms. The lateral size of the supercell was $4 \times 4$ for flat surfaces and $6 \times 4$ for step calculations. Atomic pseudopotentials were generated according to Ref. [19] and the Brillouin zone summations were approximated by one mean-value $k$ point.

[19] D. R. Hamann, Phys. Rev. B 40, 2980 (1989).

[20] C. Wang, Q.-M. Zhang, and J. Bernholc, Phys. Rev. Lett. 69, 3789 (1992).

[21] P. Bogusławski et al., Phys. Rev. Lett. 72, 3694 (1994), and references therein.

[22] Similar results were obtained by J.-H. Cho, S. Jeong, and M.-H. Kang, Phys. Rev. B 50, 17139 (1994). These authors have considered the $2 \times 1$ reconstruction with $1 / 2 \mathrm{ML}$ of Ge on Si. Here, we confirm their results for the realistic $c(2 \times 4)$ reconstruction.

[23] X. Chen et al., Phys. Rev. B 55, R7319 (1997); R. Larciprete et al., ibid. 61, 16006 (2000).

[24] D. J. Chadi, Phys. Rev. Lett. 59, 1691 (1987).

[25] P. Bogusławski, in Proceedings of the 21st International Conference on the Physics of Semiconductors, Beijing, China, 1992, edited by P. Jiang and H. Zheng (World Scientific, Singapore, 1993), p. 397.

[26] G. Brocks, P. J. Kelly, and R. Car, Phys. Rev. Lett. 66, 1729 (1991); Q.-M. Zhang, C. Roland, P. Bogusławski, and J. Bernholc, Phys. Rev. Lett. 75, 101 (1995). 